\title{
Full evolutionary models for PG 1159 stars. Implications for the helium-rich $\mathrm{O}(\mathrm{He})$ stars
}

\author{
M. M. Miller Bertolami ${ }^{1,2,3, \star}$ and L. G. Althaus ${ }^{1,2, \star \star}$ \\ 1 Facultad de Ciencias Astronómicas y Geofísicas, Universidad Nacional de La Plata, Paseo del Bosque S/N, \\ B1900FWA La Plata, Argentina \\ e-mail: [mmiller;althaus]@fcaglp.unlp.edu.ar \\ 2 Instituto de Astrofísica La Plata, IALP, CONICET-UNLP, Argentina \\ 3 Max-Planck-Institut für Astrophysik, Garching, Germany
}

Received 19 December 2005 / Accepted 7 March 2006

ABSTRACT

\begin{abstract}
Aims. We present full evolutionary calculations appropriate to post-AGB PG 1159 stars for a wide range of stellar masses. Methods. We take into account the complete evolutionary stages of PG 1159 progenitors starting from the Zero Age Main Sequence. We consider the two kinds of Born Again Scenarios, the very late thermal pulse (VLTP) and the late thermal pulse (LTP), that give rise to hydrogen-deficient compositions. The location of our PG 1159 tracks in the effective temperature-gravity diagram and their comparison with previous calculations as well as the resulting surface compositions are discussed.

Results. Our results reinforce the idea that the different abundances of ${ }^{14} \mathrm{~N}$ observed at the surface of those PG 1159 stars with undetected hydrogen is an indication that the progenitors of these stars would have evolved through a VLTP episode, where most of the hydrogen content of the remnant is burnt, or LTP, where hydrogen is not burnt but instead diluted to very low surface abundances. We derive new values for spectroscopical masses based on these models. We discuss the correlation between the presence of planetary nebulae and the ${ }^{14} \mathrm{~N}$ abundance as another indicator that ${ }^{14} \mathrm{~N}$-rich objects should come from a VLTP episode while ${ }^{14} \mathrm{~N}$-deficient ones should be the result of a LTP. Finally, we discuss an evolutionary scenario that could explain the existence of PG 1159 stars with unusually high helium abundances and a possible evolutionary connection between these stars and the low mass $\mathrm{O}(\mathrm{He})$ stars.
\end{abstract}

Key words. stars: evolution - stars: AGB and post-AGB - stars: interiors

\section{Introduction}

It is accepted that the hot, luminous PG 1159 stars constitute the immediate descendants of post-asymptotic giant branch (postAGB) evolution. They provide an evolutionary link between these stars and most of the hydrogen $(\mathrm{H})$-deficient white dwarf (WD) stars. PG 1159 are thought to be formed via a born again scenario, that is, a very late thermal pulse (VLTP) experienced by a hot WD during its early cooling phase (Fujimoto 1977; Schönberner 1979; Iben et al. 1983, for earlier references) or a late thermal pulse (LTP) that occurs during the post-AGB evolution when $\mathrm{H}$ burning is still active (see Blöcker 2001 for references). During the VLTP, an outward-growing convection zone powered by the helium burning shell develops and reaches the $\mathrm{H}$-rich envelope of the star, with the consequence that most of the hydrogen content is burnt. The star is then forced to evolve rapidly back to the AGB and finally into the central star of a planetary nebula at high effective temperatures $\left(T_{\text {eff }}\right)$ but now as a H-deficient, quiescent helium-burning object. LTP also leads to a hydrogen-deficient composition but as a result of a dilution episode. Consequently, while post-VLTP models are expected to become H-depleted (DB) white dwarfs (Althaus et al. 2005a), it has been argued that post-LTP models may finally (after the PG 1159 stage) evolve into H-rich (DA) WDs with thin H envelopes (Althaus et al. 2005b).

\footnotetext{
* Fellow of CONICET, Argentina.

$\star \star$ Member of the Carrera del Investigador Científico y Tecnológico and IALP, CONICET / FCAG-UNLP, Argentina.
}

Currently, 37 stars are members of the PG 1159 family, which span a wide domain in the $\log g-T_{\text {eff }}$ diagram: $5.5 \lesssim$ $\log g \lesssim 8$ and $75000 \mathrm{~K} \lesssim T_{\text {eff }} \lesssim 200000 \mathrm{~K}$ (see Werner \& Herwig 2006 for a review). Roughly half of them are still embedded in a planetary nebula. A striking feature characterizing PG 1159 stars is their peculiar surface chemical composition. Indeed, spectroscopic analyses have revealed that most PG 1159 stars exhibit H-deficient and helium-, carbon- and oxygen-rich surface abundances. Surface mass abundances of about $0.33 \mathrm{He}, 0.5 \mathrm{C}$ and $0.17 \mathrm{O}$ are reported as typical, though notable variations are found from star to star (Dreizler \& Heber 1998; Werner 2001). In fact, almost every star has an individual $\mathrm{He} / \mathrm{C} / \mathrm{O}$ mixture. According to Werner (2001) helium abundances range between $33-85 \%$ and carbon and oxygen abundances span the ranges $13-58$ and $2-24 \%$, respectively. The rich variety of surface patterns observed in PG 1159 stars poses a challenge to the theory of post-AGB evolution. In particular, the appreciable abundance of oxygen in the atmospheres of these stars has been successfully explained by Herwig et al. (1999) on the basis of evolutionary calculations of the born-again scenario that incorporate convective overshoot. In addition, hydrogen has been detected in some PG 1159s (called hybrid-PG 1159 stars). These H-rich PG 1159s are currently interpreted in terms of a final helium shell flash experienced by the progenitor stars immediately before their departure from the AGB (AGB final thermal pulse or AFTP scenario, Herwig 2001). Interest in PG 1159 stars is also motivated by the fact that about ten of them exhibit multiperiodic luminosity variations caused by non-radial $g$-mode 
Table 1. Times for different moments of the evolution of the PG 1159 progenitors (in $10^{6} \mathrm{yr}$ since the ZAMS). The column below $t_{\mathrm{CHeB}}$ marks the times at the start and the end of core helium burning $(\mathrm{CHeB})$. Also the number of thermal pulses is given (Col. 5).

\begin{tabular}{cccccc}
\hline \hline Initial mass & $\Delta t_{\mathrm{MS}}$ & $t_{\mathrm{CHeB}}$ & $t_{\mathrm{TP}-\mathrm{AGB}}$ & \#TP & Final mass [in $M_{\odot}$ ] \\
\hline $1 M_{\odot}$ & 9790 & $12666-12790$ & 12804 & $5 / 7$ & $0.530 / 0.542$ \\
$2.2 M_{\odot}$ & 879 & $904-1158$ & 1183 & 16 & 0.565 \\
$2.5 M_{\odot}$ & 595 & $609-818$ & 832 & 13 & 0.584 \\
$3.05 M_{\odot}$ & 374 & $380-473$ & 480 & 11 & 0.609 \\
$3.5 M_{\odot}$ & 254.5 & $257.5-314$ & 319 & 8 & 0.664 \\
$5.5 M_{\odot}$ & 81 & $81.6-92.5$ & 93.3 & 8 & 0.870 \\
\hline
\end{tabular}

pulsations, a fact that has attracted the attention of researchers (see Quirion et al. 2004; Gautschy et al. 2005; Córsico \& Althaus 2006 for recent works).

Many relevant aspects of PG 1159 stars, for instance mass determinations, precise asteroseismological fits, pulsation stability analysis and ages call for extensive full evolutionary models of PG 1159 stars with different stellar masses and realistic histories that led them through the thermally pulsing AGB and born again phases (see Dreizler \& Heber 1998; Werner 2001). The lack of a grid of evolutionary sequences with different masses in the literature has motivated us to undertake the present work, which is aimed at providing full evolutionary tracks appropriate for PG 1159 stars based on a complete and selfconsistent treatment of the progenitor stars, starting from the main sequence. Specifically, we follow the evolution of initially 1, 2.2 2, 2.5, 3.05, 3.5 and $5.5 M_{\odot}$ stars from the zero-age main sequence (ZAMS) through the thermally pulsing and mass-loss phases on the AGB to the PG 1159 regime. In particular, the evolutionary stages corresponding to the almost complete burning of protons following the occurrence of the VLTP and the ensuing born-again episode are carefully followed for each sequence. In addition we have followed the evolution of initially 2.2 and $2.7 M_{\odot}$ model stars through the surface hydrogen dilution phase that follows the LTP. Attention is paid to the abundance changes during the entire evolution, which are described by means of a time-dependent scheme for the simultaneous treatment of nuclear evolution and various mixing processes. We also develop an evolutionary scenario to address the existence of PG 1159 stars with unusually high helium abundances and nitrogen deficiency, an issue recently raised by Quirion et al. (2004) in the context of pulsating PG 1159s. We also apply this evolutionary scenario to address the existence of low-mass $\mathrm{O}(\mathrm{He})$ stars.

The paper is organized as follows. The following section contains the main physical inputs to the models and a description of the evolutionary sequences considered. In Sect. 3 we present the evolutionary results. There, we elaborate on the main aspects of pre-PG 1159 evolution, particularly during the bornagain phase and the attendant chemical changes. In this section we also compare our PG 1159 models with those existing in the literature. In Sect. 4, we discuss the implications of our results. We also discuss an evolutionary scenario that could explain the existence of PG 1159s with high helium content. Section 5 is devoted to summarizing our results.

\section{Input physics and evolutionary sequences}

The calculations presented in this work have been done with the LPCODE evolutionary code appropriate for computing the formation and evolution of WD stars through late thermal pulses (Althaus et al. 2005a,b). LPCODE uses OPAL radiative opacities (including carbon- and oxygen-rich compositions) from the compilation of Iglesias \& Rogers (1996), complemented, at low temperatures, with the molecular opacities from
Alexander \& Ferguson (1994). In this work we adopt solar metallicity. The abundance evolution of 16 chemical elements is included via a time-dependent numerical scheme that simultaneously treats nuclear evolution and mixing processes due to convection, salt fingers and overshooting (OV). Such a treatment is particularly important during the short-lived phase of the VLTP and the ensuing born-again episode. Computationally, convective OV was treated as an exponentially decaying diffusive process above and below any formally convective region, including the convective core (main sequence and central helium burning phase), the helium flash convection zone and the external convective envelope (see Herwig et al. 1999, for details).

We present six model star sequences, with initially $1,2.2$, $2.5,3.05,3.5,5.5 M_{\odot}$. All of the sequences are followed from the ZAMS through the thermally pulsing and mass loss phases on the AGB (TP-AGB). After experiencing several thermal pulses (see Table 1 for a description of the evolution of the sequences up to the TP-AGB), the progenitors depart from the AGB and evolve towards high $T_{\text {eff }}$. Mass loss during the departure from the AGB has been arbitrarily set, until $\log \left(T_{\text {eff }}\right) \sim 4$ when it was turned off, so to obtain a final helium shell flash during the early WD cooling phase.

The $1 M_{\odot}$ model experienced a core helium flash, which led it to the horizontal branch to burn helium in a stable fashion. For this sequence two different AGB evolutions have been considered, with different mass loss rates so to obtain a different number of thermal pulses and, eventually, two different remnant masses of 0.53 and $0.542 M_{\odot}$. After mass loss episodes, the stellar masses of the post-AGB models is reduced to $0.53,0.542$, $0.565,0.584,0.609,0.664$ and 0.87 . Except for the $1 M_{\odot}$ sequences we find convergence problems shortly after the departure from the AGB, probably due to the lack of a hydrostatic solution in our models (see Wood \& Faulkner 1986), problems that are more noticeable when OPAL opacities are used. To overcome this difficulty we have temporarily increased the mixing length theory parameter $\alpha$ by about a factor of two during the early departure from the AGB $\left(\log T_{\text {eff }} \lesssim 3.6\right)$. For the purpose of the present work, this artificial procedure does not bear any relevance for the further stages of evolution. Our most massive model $\left(0.870 M_{\odot}\right)$ experienced several convergence problems, in the envelope and atmosphere integration, due to the very high radiation pressure that characterizes the outer layers of this model. This causes the density of the hydrostatic model to drop to almost zero and thus our sequence failed to converge. To continue with evolution, we imposed artificial boundary conditions during its redward excursion after the VLTP. We particularly checked that this modification did not alter the location of the model in the $\log g-T_{\text {eff }}$ diagram by doing the same modification to the $3.5 M_{\odot}$ model, for which the evolution after the VLTP was consistently calculated, and we find that no effect of this artificial evolution was present when the model reached the PG 1159 phase. 


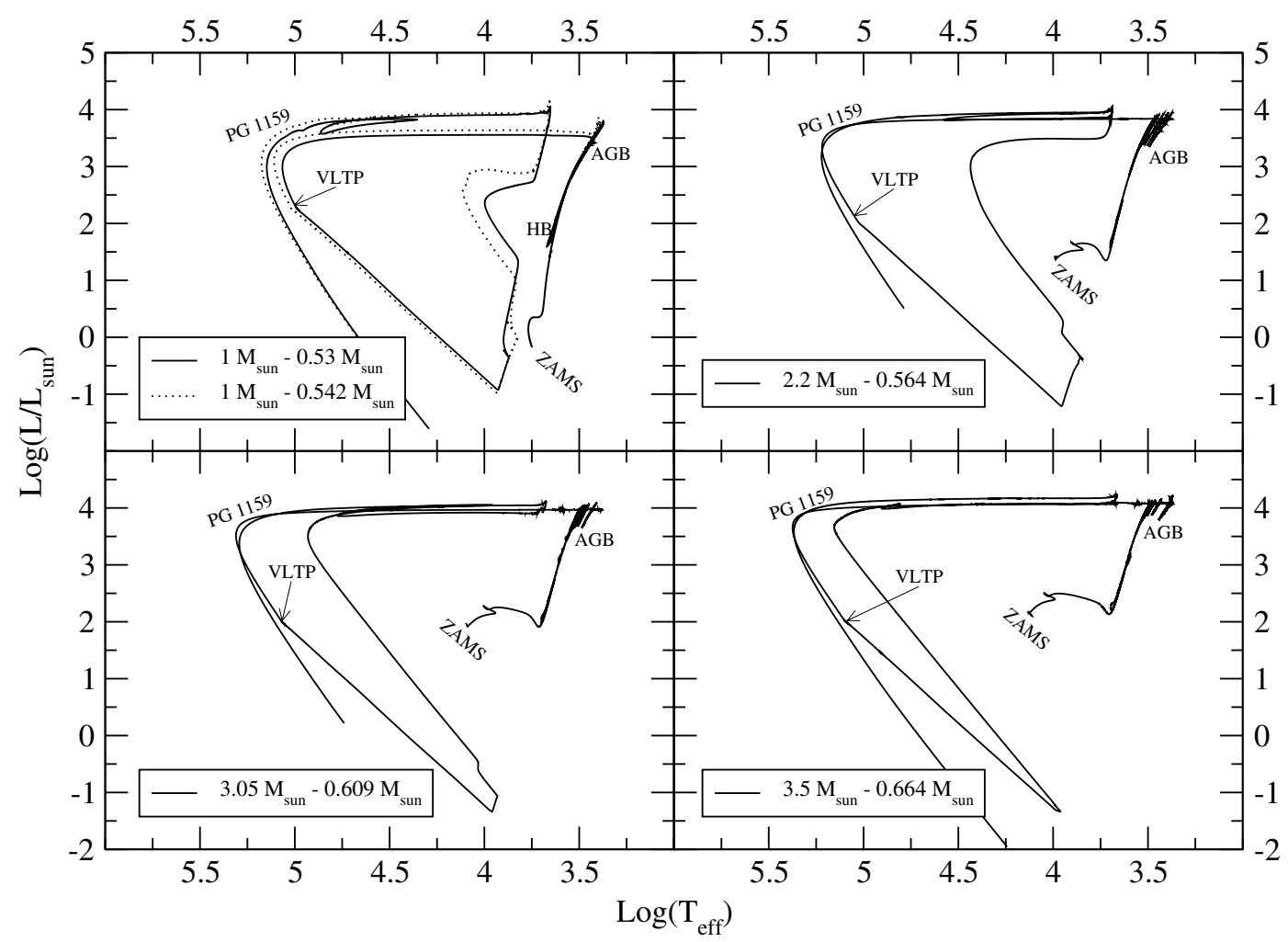

Fig. 1. HRDs for selected VLTP sequences. Legends indicate initial and final masses of the models. In the upper left panel, the dotted line corresponds to the case in which a larger number of thermal pulses was allowed before the progenitor departs from the AGB.

\section{Evolutionary results}

\subsection{Evolution during the born-again phase}

In this work we do not intend to present a detailed study of the born again phase leading to PG 1159 stars, an aspect which will be deferred to a later publication. Instead we limit our discussion to presenting the main characteristics of the evolution of the progenitor stars relevant for PG 1159s. In Fig. 1 we show the complete Hertzsprung-Russell diagram (HRD) for four of our calculated sequences (for a complete discussion of the evolution of an initially $2.5 M_{\odot}$ sequence see Miller Bertolami et al. 2006). As can be noted, our numerical simulation covers all the evolutionary phases from the ZAMS to the domain of the earlyWD including the stages corresponding to the helium thermal pulses at the tip of the AGB and the born again episode. This last stage is particularly fast and evolution proceeds on a scale of years (Herwig 2001; Miller Bertolami et al. 2006). During this stage the star is ballooned back to the giant region both by the helium flash and by the violent H-burning that occurs at the VLTP. Note that the detailed evolution of the sequences during this stage depends on the mass of the remnant. In particular the expansion caused by H-burning is very dependent on the mass of the model. We find that the double loop path in the HR, characteristic of successive $\mathrm{H}$ - and He-driven expansions (Lawlor \& MacDonald 2003), is only present for intermediate mass models $\left(0.55 M_{\odot} \lesssim M \lesssim 0.61 M_{\odot}\right)$. This is because for models with lower masses the H-driven expansion is strong enough to keep the model at low effective temperatures until the effect of the He-driven expansion takes over, so that no return to the blue after the H-driven expansion happens. On the other hand, for massive models we find that H-driven expansion is extremely weak and it is almost suppressed (it stops at $\log \left(T_{\text {eff }}\right) \lesssim 4.75$ ), see Fig. 1.
The effective temperature at which the model emerges at high luminosities is a function of the stellar mass. As can be seen in Fig. 1 we find that the lower the mass of the model, the lower the effective temperature at which the model becomes bright (from $\log \left(T_{\text {eff }}\right) \sim 4$ for the $0.53 M_{\odot}$ to 5.1 for the $0.664 M_{\odot}$ ).

In Fig. 2 we show chemical abundances (abundances throughout this article are given in mass fractions) throughout the interior of most of the models presented in this work (including the previously calculated $2.5 M_{\odot}$ ) at the moment when the models have attained high luminosities after the VLTP. Note the dependance of the composition profile on the stellar mass. The different envelope compositions are due partly to the fact that each sequence underwent a different number of thermal pulses (see Table 1) during the AGB phase ${ }^{1}$. The abundance profiles shown in Fig. 2 correspond to evolutionary stages well before the occurrence of the envelope mixing by convection and mass loss episodes that take place at very low temperatures after the VLTP. Consequently the depicted surface abundances of these models are not strictly the ones expected in PG $1159 \mathrm{~s}^{2}$. As can be seen in Fig. 2 after the VLTP models show a chemical structure that can be summarized as follows: a $\mathrm{C}-\mathrm{O}$ core, an intershell region rich in $\mathrm{He}, \mathrm{C}, \mathrm{O}$ and a surrounding envelope where also high amounts of ${ }^{13} \mathrm{C}$ and ${ }^{14} \mathrm{~N}$ (created at the VLTP) are found. We show the chemical stratification prior to the envelope

1 We find that the intershell helium abundance reaches a local minimum value after the third-seventh (depending on stellar mass) thermal pulse (see also Fig. 11 in Herwig 2000).

2 Because these abundances should be the consequence of both the envelope dilution and mass loss. We note that dilution of the envelope material with the intershell layers leads to lower ${ }^{14} \mathrm{~N}$ surface abundances, between 0.005 and 0.025 by mass which is similar to the observed ones (Miksa et al. 2002). 


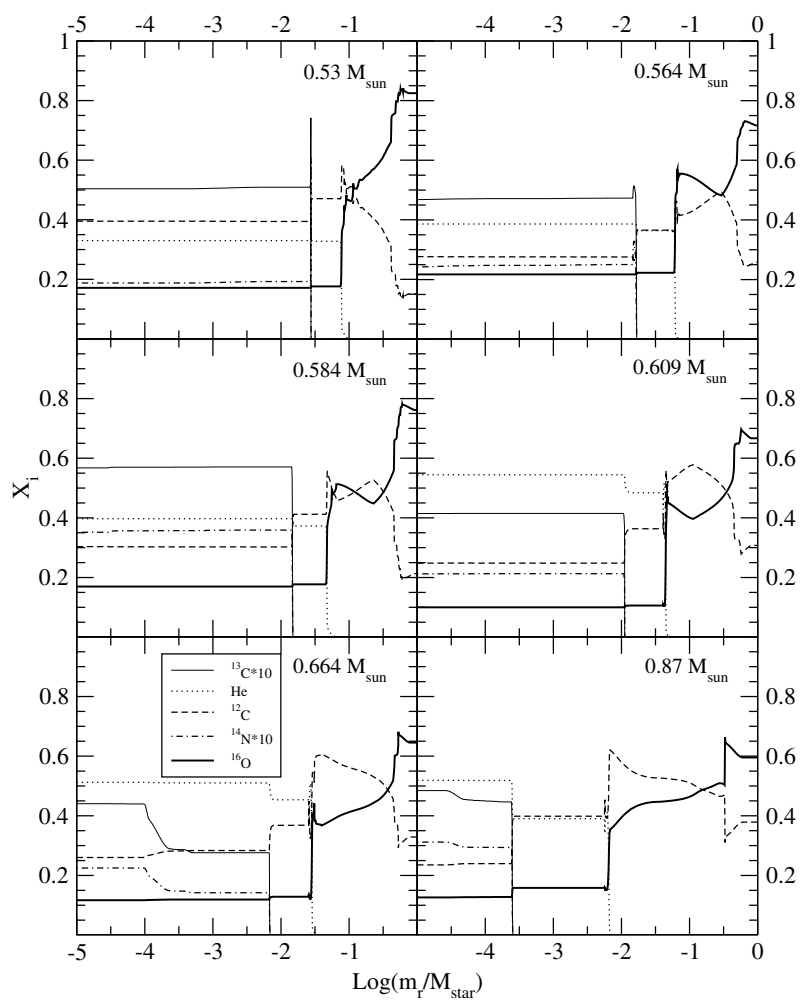

Fig. 2. Internal chemical profiles for VLTP models having different stellar masses shortly after the star has attained high luminosities after the VLTP. These chemical profiles will be affected by the envelope mixing and mass loss episodes when models return to the domain of the giant region. Abundances are given in mass fractions.

mixing and mass loss episodes because both the deepness of the envelope convection after the VLTP and the mass loss rate are highly uncertain and will affect the final surface composition. For example all of our models remain at $\log \left(T_{\text {eff }}\right) \lesssim 3.8^{3}$ for about 700-800 yr. Mass loss rates similar to those observed in the Sakurai's object $\left(1 \times 10^{-5} M_{\odot} / \mathrm{yr}\right.$, and even higher, see Hajduk et al. 2005) would cause $7 \times 10^{-3} M_{\odot}$ to be stripped off from the star ${ }^{4}$. In all the cases the expected surface abundances should be somewhat between those displayed by models in the envelope and at the intershell region (Fig. 2). The helium abundance is particularly noteworthy. Indeed note that our postVLTP models predict that the final surface abundance of helium will be in the range $0.3-0.55$ by mass, which is in agreement with the range of observed helium abundance in most PG 1159s (see Werner \& Herwig 2006). However these abundances should be taken with caution as they are not only determined by the stellar mass but also by the number of pulses considered in the AGB stage and the exact value of the adopted OV parameter $(f)$ (Herwig 2000).

\subsection{PG 1159 stage: overall location of our models in the $\log g-T_{\text {eff }}$ diagram}

In this section we focus on the location of the models in the $\log g-T_{\text {eff }}$ diagram. We compare them with the available data from observations and from previous stellar models for

\footnotetext{
${ }^{3}$ Except for the $0.870 M_{\odot}$ for which we cannot obtain reliable ages.

4 This strong mass loss at low effective temperatures may help to explain the bizarre surface abundance of $\mathrm{H} 1504+65$, which is difficult to explain by only considering mass loss during the standard post-AGB evolution (Schönberner \& Blöcker 1992).
}

H-deficient post-AGB stars (Figs. 3 and 4 respectively). As can be seen in Fig. 4 our models are somewhat hotter than previous models of He-burning objects with $\mathrm{H}$-deficient surface abundances. From visual inspection one can conclude that the mean shift in spectroscopical masses due to this difference in the surface temperatures would be $0.025 M_{\odot}$ for O’Brien \& Kawaler (as presented in Dreizler \& Heber 1998) and $0.04 M_{\odot}$ for Wood \& Faulkner models (Wood \& Faulkner 1986). Also note (Fig. 3) the existence of H-deficient objects that are cooler than our less massive sequences. Although typical uncertainties in the derived values of $g$ and $T_{\text {eff }}$ for PG 1159 stars prevent us from drawing conclusions, this discrepancy may be indicating that our sequences are somewhat hotter than real stars. On the other hand, we find our models to be in good agreement with the 0.604 track presented by Werner \& Herwig (2006), which is based on similar physical assumptions. This seems confirm the finding by these authors that the location of the models in the $\log g-T_{\text {eff }}$ diagram is very sensitive to the modeling of the previous AGB evolution. In particular Werner \& Herwig (2006) point out that this difference may be related with the effect of a smaller effective core growth caused by the more efficient third dredge-up events of the new models (mainly as consequence of the inclusion of overshooting mixing at the He-flash driven convection zone during the TP-AGB). If this is true, a lower $f$-value than adopted in the present article $(f=0.016)$ at the He-flash driven convection zone would lead to a less efficient third dredge-up and, thus, would shift post-AGB tracks to lower effective temperatures ${ }^{5}$. This speculation would be in line with hydrodynamical simulations of the He-shell flash (Herwig et al. 2006) that suggest that OV may be reduced to a minimum. However, it should be kept in mind that a quite efficient overshooting at the base of the shell flash driven convective zone (during the TP-AGB) is needed in order to obtain oxygen surface abundances like those displayed by PG 1159 stars (Herwig et al. 1999).

For the location of post-AGB tracks in the $\log g-T_{\text {eff }}$ diagram, previous works (Werner 2001) have noted that it was possible that spectroscopic masses that are based on models with normal surface abundances may suffer from systematic errors. As it is shown in Fig. 5 H-burning models are indeed different from those which are He-burning of similar mass. However the effect is not the same for all masses. We find that differences are larger for models of lower mass. Our H-burning model of $0.53 M_{\odot}$ is located at much lower temperatures than the He-burning model of similar mass (and thus closer to the location of the low mass models of Blöcker 1995). Another difference is that in the high-g side of the knee, on the $\log g-T_{\text {eff }}$ diagram, H-burning models are systematically less compact.

\section{Discussion}

\subsection{Are PG 1159 stars post VLTP or LTP stars?}

All of our VLTP-models display high amounts of ${ }^{14} \mathrm{~N}$ at their surface (and also a high ${ }^{13} \mathrm{C} /{ }^{12} \mathrm{C}$ ratio see Fig. 2). This is similar to the result of Iben \& MacDonald (1995), but at variance with later results for the VLTP by Herwig (2001). Since many PG 1159s do not exhibit ${ }^{14} \mathrm{~N}$ (Dreizler \& Heber 1998), we analyze other possible channels for the formation of PG 1159 stars which do not yield high ${ }^{14} \mathrm{~N}$ surface abundances. We look for changes in the location of the models in the $\log g-T_{\text {eff }}$ diagram

\footnotetext{
${ }^{5}$ After the acceptance of this paper we have not found (in preliminary calculations) any important shift for remnants of similar mass but different effective core growths during the TP-AGB. We intend to explore this effect in a future work.
} 


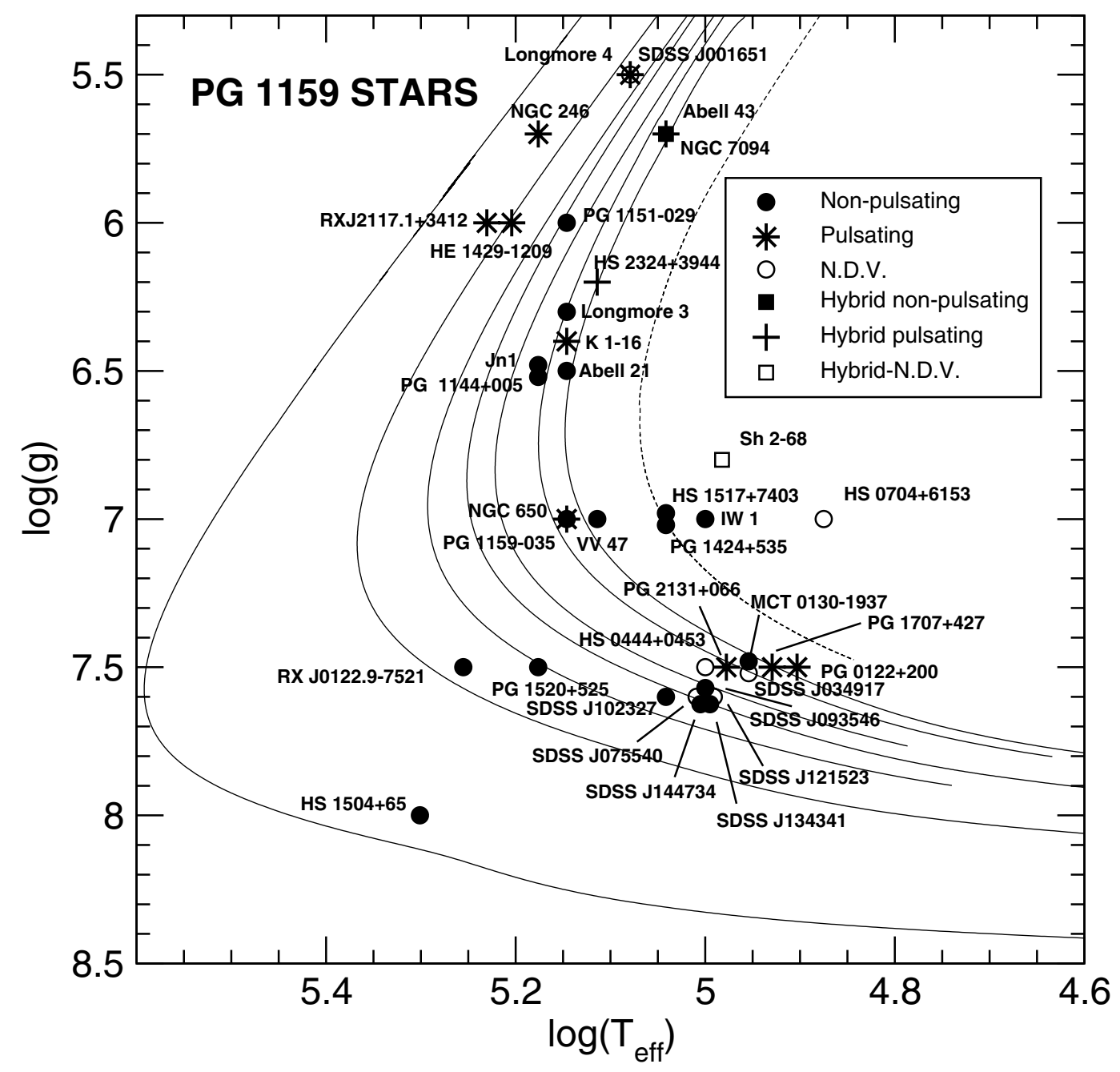

Fig. 3. Location of analyzed PG 1159 stars (values taken from Werner \& Herwig 2006). Empty symbols are used for the objects for which no information about their variability is available (N.D.V.). Solid lines correspond to our evolutionary sequences $0.87,0.664,0.609,0.584,0.564$, 0.542 and $0.53 M_{\odot}$. The broken line corresponds to the (helium enriched) $0.512 M_{\odot}$ post early AGB model discussed in Sect. 4.2.

if PG 1159 models were the offspring of a LTP, in which H is not burnt but diluted after the helium flash and thus no enhanced ${ }^{14} \mathrm{~N}$ abundance is expected. Consequently we carried out two additional calculations of sequences that went through a LTP (of 0.589 and $0.562 M_{\odot}$ ). These are shown in Fig. 6 where their location in the $\log g-T_{\text {eff }}$ diagram can be seen compared to VLTP sequences of similar mass. There is only a slight difference between VLTP and LTP tracks that occurs in the low-gravity region before reaching the knee ${ }^{6}$. This may be connected to the fact that, there, the H-burning shell is still active in LTP sequences.

In both LTP-sequences, $\mathrm{H}$ was diluted to about a $4 \%$ in mass $^{7}$, which is close to the detection limit (Werner 2001). Our LTP-models do not display enhanced ${ }^{14} \mathrm{~N}$ abundance at their surface. Our results reinforce the idea of Werner et al. (1998) that the different abundances of ${ }^{14} \mathrm{~N}$ in the PG 1159 sample seems to be an indication that PG 1159 progenitors are a mixture of VLTP and LTP-AFTP cases. As was mentioned by Werner (2001) the

${ }^{6}$ However while our models are calculated adopting solar values for the heavy metal abundances, it is known that post-VLTP stars display a non-solar heavy metal distribution (Asplund et al. 1999; Miksa et al. 2002). For example, we note that tracks move 0.03 dex to the right (red) if opacities are increased by $\sim 40 \%$.

$7 \mathrm{He} / \mathrm{C} / \mathrm{O}$ abundances are $31 / 40 / 20$ and $33 / 36 / 21$ for the 0.589 and $0.562 M_{\odot}$ models, respectively. existence of LTP progenitors in the PG 1159 sample may help to solve the inconsistency presented by the expansion ages of planetary nebulae. Therefore we discuss the presence of planetary nebulae with regard to the location of their central stars in the $\log g-T_{\text {eff }}$ diagram and their corresponding evolutionary ages. In Fig. 7 we draw isochrones based on the evolutionary ages of our models. We note that ages are measured since the moment of the VLTP and not since the first departure from the $\mathrm{AGB}^{8}$.

In order to compare theory with observations, and to distinguish between stars coming from VLTP, LTP and AFTP scenarios, we examine how observable parameters such as the presence of planetary nebulae, surface abundances and pulsational instability correlate with each other as well as how the presence of high ${ }^{14} \mathrm{~N}$ surface abundances correlates with the existence of planetary nebulae around PG 1159 stars. We will discuss the implications of our evolutionary sequences in the light of the correlation between the presence of ${ }^{14} \mathrm{~N}$ and pulsations found by Dreizler \& Heber (1998). We will assume that this correlation is valid for all PG 1159 non-pulsators within the instability strip (however see Werner \& Herwig 2006 for a redetermination of nitrogen, 0.001 by mass, in the pulsating PG 1159-035), even though this correlation is based on a very small sample (9 stars).

\footnotetext{
8 Also note that, as no mass loss has been considered in these sequences after the VLTP, these values should be taken as upper limits.
} 


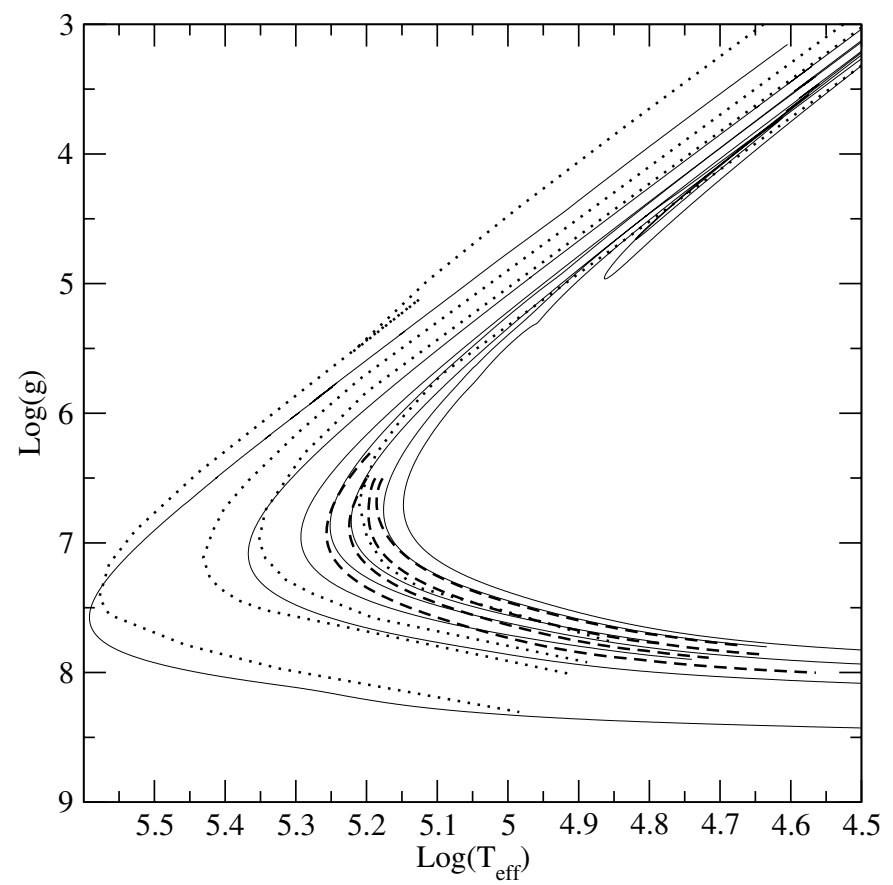

Fig. 4. Comparison of the models presented in this work (thin lines; $0.87,0.664,0.609,0.584,0.565,0.542,0.53 M_{\odot}$ ) with previous models of He-burning objects. Dotted lines correspond to Wood \& Faulkner 1986 type B mass loss models $\left(0.87,0.76,0.7,0.6 M_{\odot}\right)$ and broken lines indicate O'Brien \& Kawaler tracks for models with PG 1159 like compositions $\left(0.63,0.6,0.573,0.55 M_{\odot}\right)$. As can be seen our models are generally hotter than previous models of similar mass.

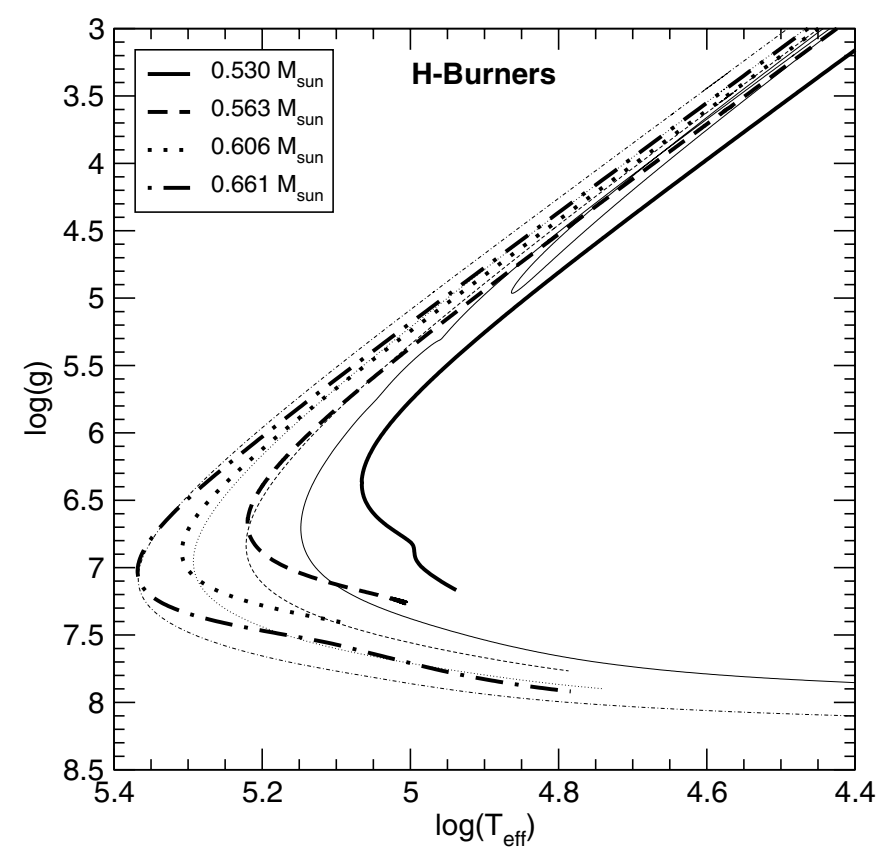

Fig. 5. Comparison of the location of $\mathrm{H}$ and He burning post AGB models. Note that at lower $g$-values (during the WC stage) H-burner are located at slightly higher gravities, while at higher gravities (beyond $\log g \sim 6.5$ ) H-burners have lower gravities than He burning objects. The low mass H-burning object lies at lower temperatures than the He-burning object of similar mass $\left(0.53 M_{\odot}\right)$.

Although ${ }^{14} \mathrm{~N}$ has been demonstrated by Quirion et al. (2004) to be unimportant in driving pulsations, ${ }^{14} \mathrm{~N}$ is an indicator of previous evolution. Stars with and without ${ }^{14} \mathrm{~N}$ are expected to have

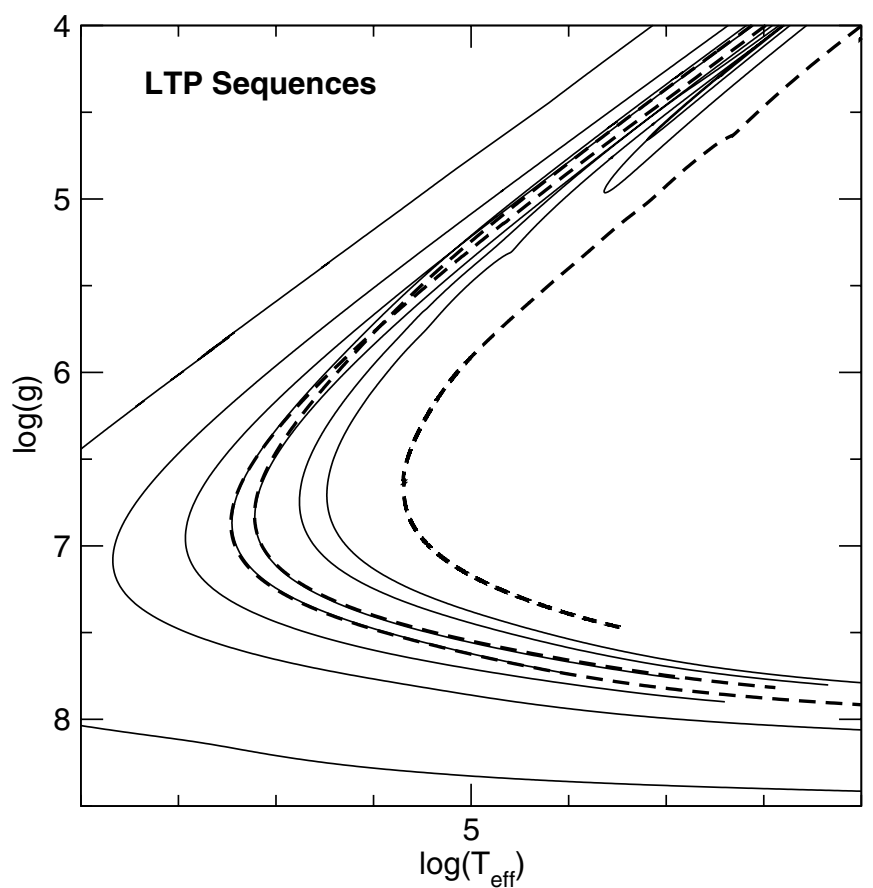

Fig. 6. LTP sequences calculated for this work $\left(0.589 M_{\odot}, 0.564 M_{\odot}\right.$, broken lines) are shown against VLTP sequences (thin lines). Also, the helium enhanced post early AGB sequence with $0.512 M_{\odot}$ (which is also a post-LTP model) is shown (coolest sequence, also in broken lines). Note that LTP and VLTP models of similar mass follow almost the same track.

different $\mathrm{H}$ and heavy elements abundances and consequently different opacities (see Iglesias et al. 1995). As the first time to the high temperature region of the HR (after departing from the AGB) takes about 10000-20000 yr (VLTP, Blöcker 1995) for low mass stars, the presence of planetary nebulae around some PG 1159s close to the isochrones of 25000 and $50000 \mathrm{yr}$ clearly implies that these objects (particularly NGC 650, VV47 and PG 1520+525) cannot be the offspring of a VLTP (because planetary nebulae should have faded during this time interval, 30000-75000 yr). This speculation seems to be corroborated by the fact that one of these objects (PG 1520+525) is known to be ${ }^{14} \mathrm{~N}$-deficient, and also by the fact that the rest of them are non-pulsating objects which are close to, or inside, the instability strip (and thus according to Dreizler \& Heber 1998 must be ${ }^{14} \mathrm{~N}$-deficient). Some stars (between the 25000 and $100000 \mathrm{yr}$ isochrone) with detected nitrogen (the ones that should be coming from a VLTP episode, for instance PG 1144+005) do not show an associated planetary nebula, whilst NGC 650 and VV45 (both non-pulsating objects) do show planetary nebulae. This correlation between the presence of ${ }^{14} \mathrm{~N}$ and the absence of the planetary nebula, seems to be another indicator that ${ }^{14} \mathrm{~N}$-rich objects should come from a VLTP while ${ }^{14} \mathrm{~N}$-deficient should come from a LTP.

This argument is not affected by the possibility that the pulsating PG 1159 at relatively low $g(\sim 6)$ (which do display planetary nebulae) may be ${ }^{14} \mathrm{~N}$ rich according to the correlation found by Dreizler \& Heber (1998). Because all these objects are more massive than $\sim 0.6 M_{\odot}$ according to our models, these objects quickly reach the WD cooling track where they experience the VLTP ( 5000 yr, see Blöcker 1995). Then, as these objects are still close to the $2000 \mathrm{yr}$ isochrone, no more than $\sim 7000 \mathrm{yr}$ have past since their first departure from the AGB and thus they can 


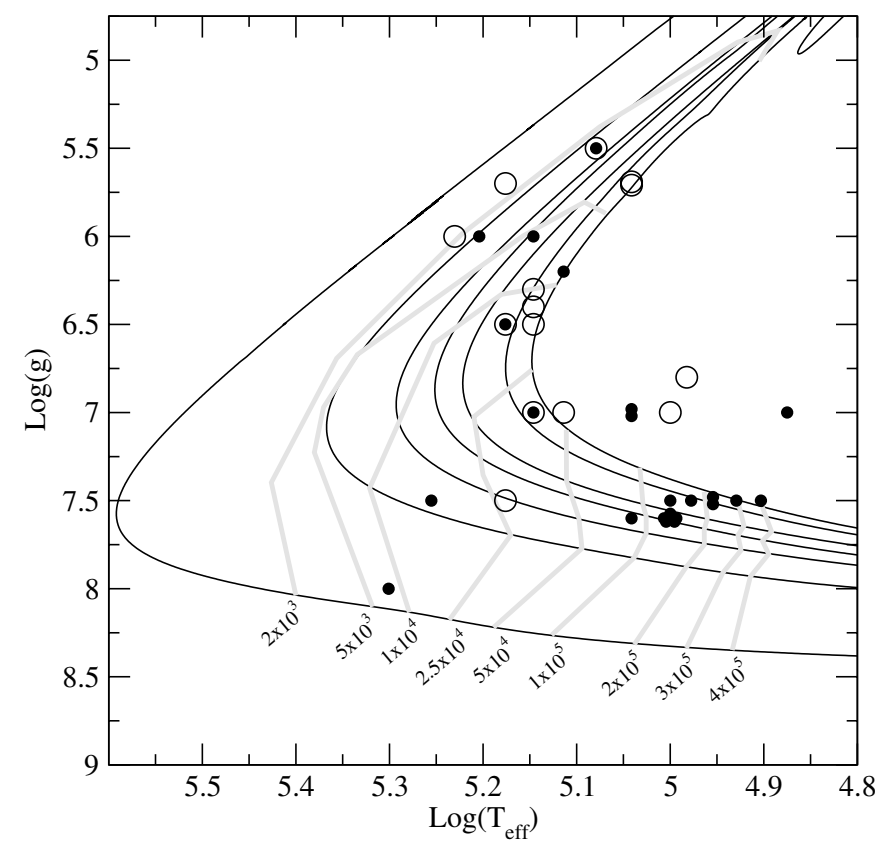

Fig. 7. Isochrones for post-VLTP sequences. Times are measured since the last helium flash. Empty circles (black dots) indicate PG 1159 stars with (without) planetary nebulae. Note the existence of some objects beyond the $2.5 \times 10^{4} \mathrm{yr}$ isochrone that show planetary nebulae. These objects could not be the result of a VLTP episode as their surrounding nebulae would have faded. Indeed, in none of these objects have significant amounts of ${ }^{14} \mathrm{~N}$ (a signature of previous proton burning during the VLTP) been found while many of them are known to be ${ }^{14} \mathrm{~N}$-deficient (Dreizler \& Heber 1998). Also note the fast evolution of the most massive model before it reaches $\log g \sim 8$, which easily explains the absence of detected PG 1159 stars with $\log T_{\text {eff }}>5.4$.

still retain their planetary nebulae, even if they have suffered a VLTP.

Even beyond the speculation about the correlation between ${ }^{14} \mathrm{~N}$ and pulsations, PG $1144+005$ and PG 1159-035, which do not show associated planetary nebulae (while in the $\log g-T_{\text {eff }}$ diagram they are surrounded by stars that do show associated planetary nebulae) are known to display ${ }^{14} \mathrm{~N}$ at their surfaces. On the other hand, PG $1520+525$ which is located closer to the same isochrone than PG $1159-035$ and that is known to be ${ }^{14} \mathrm{~N}$ deficient $\left(N \lesssim 1 \times 10^{-4}\right.$, Miksa et al. 2002), does show a planetary nebula.

In this section we mentioned that the existence of two groups of ${ }^{14} \mathrm{~N}$-abundances in PG 1159 stars could be explained by the existence of two different evolutionary channels leading to the PG 1159 stage (namely VLTP and LTP episodes). If so, our models predict ${ }^{14} \mathrm{~N}$-deficiency to be directly related to the presence of $\mathrm{H}$ below the detection limit. This claim might be supported by the correlation between the presence of ${ }^{14} \mathrm{~N}$ and the absence of planetary nebulae. However, it is clearly at variance with the reported correlation between helium and ${ }^{14} \mathrm{~N}$ mentioned by Quirion et al. (2004), as there is no simple reason why LTP models only should produce heliumenriched PG 1159 stars. In the following section we will discuss an evolutionary scenario for these helium-enriched stars (namely HS 1517+7403, HS 0740+6153 and MCT 0130-1937), an explanation related to the low mass of these stars.

\subsection{Low $T_{\text {eff }}$-Low $g$ PG 1159 stars. The existence of helium-enriched PG 1159 stars and their possible connection with low mass $\mathrm{O}(\mathrm{He})$ stars}

In the previous section we assumed the fact that pulsating PG 1159s are rich in ${ }^{14} \mathrm{~N}$; however as was noted by Dreizler \& Heber (1998) and also by Quirion et al. (2004) many of the nonpulsator stars in the sample have an unusually high helium abundance. This is important in view of the results found by Quirion et al. (2004) that this helium enrichment could be the cause of the existence of non-pulsators within the instability strip ${ }^{9}$. If non-pulsators are indeed characterized by helium-enhanced, $\mathrm{N}^{14}$-deficient surface abundances they cannot be the result of the standard LTP scenario experienced by post TP-AGB stars. Also, it is clear Fig. 3 that many of the H-deficient stars lie too much to the red of the post-VLTP models presented in this work. Although comparison with observations has to be taken with care due to high uncertainties in the determination of $g$ (typical errors in $\log g$ are about $\sim 0.5$ dex), we feel that this discrepancy between models and real stars deserves some discussion. Firstly, these objects are probably not VLTP descendants, as three of them (PG 1424+535, HS 0704+6153 and HS 1517+7403) are known to be ${ }^{14} \mathrm{~N}$-deficient while no ${ }^{14} \mathrm{~N}$-enhanced PG 1159 is known in that region of the $\log g-T_{\text {eff }}$ diagram. Also as it is shown in Fig. 7 some of these objects still retain a planetary nebula, a fact that is difficult to understand within the VLTP scenario (Werner 2001). TP-AGB progenitor stars that went through a LTP is a possibility to explain the existence of these PG 1159s. However, as shown in Fig. 6, LTP and VLTP models of similar masses seem to be located at similar places in the $\log g-T_{\text {eff }}$ diagram. Thus if these stars are descendents of LTP episodes they should be objects with very low stellar masses ${ }^{10}$.

We have explored an evolutionary scenario that could explain the existence of the abovementioned PG 1159s. In addition we have found that this scenario may offer an alternative explanation for the formation of low mass $\mathrm{O}(\mathrm{He})$ stars. This subclass of H-deficient stars is characterized by the fact that their members display almost pure He-envelopes with only small traces of additional elements. They have been proposed to be the offspring of a merging event between two white dwarfs (Rauch et al. 2004, see also Saio \& Jeffery 2002 for the simulation of the merging event) and the direct descendants of $\mathrm{RCrB}$ stars. However at first glance it would seem that the final masses of the products of a merging event (Saio \& Jeffery 2002) should be higher than the masses derived for the two low mass $\mathrm{O}(\mathrm{He})$ stars (roughly $\sim 0.55 M_{\odot}$ ). In what follows we will show that the two $\mathrm{O}(\mathrm{He})$ stars with low mass (namely K 1-27 and HS 2209+8229) may be the direct progenitors of the He-enriched PG 1159 stars. In particular K1-27, for which detailed abundances have been derived, displays a surface composition typical of the He buffer of AGB stars, i.e. almost pure $\mathrm{He}$ $(\sim 98 \%)$ with traces of ${ }^{14} \mathrm{~N}(\sim 1.7 \%)$ (Werner \& Herwig 2006). Both of these stars are supposed to still have an appreciable mass loss rate of about $\sim 10^{-9} M_{\odot} / \mathrm{yr}$, as derived from radiation driven wind theory (see for example Table 4 of Rauch et al. 1998).

To analyze an evolutionary pathway that could give rise to ${ }^{14} \mathrm{~N}$-deficient, He-enhanced PG 1159 stars, we have calculated

\footnotetext{
${ }^{9}$ Note, however, that many non-pulsators in the observational instability strip are found to have standard helium abundances (Longmore 3, Abell 21, VV47, PG 1424+535 and PG 1151-029). On the other hand, NGC 246 which displays a high helium abundance $(\sim 60 \%$, Werner \& Herwig 2006) is a pulsating PG 1159.

${ }^{10}$ However it should be noted that stars with very low mass may need an unacceptably long time to reach the PG 1159 stage ( $>12.8 \mathrm{Gyr}$ ).
} 


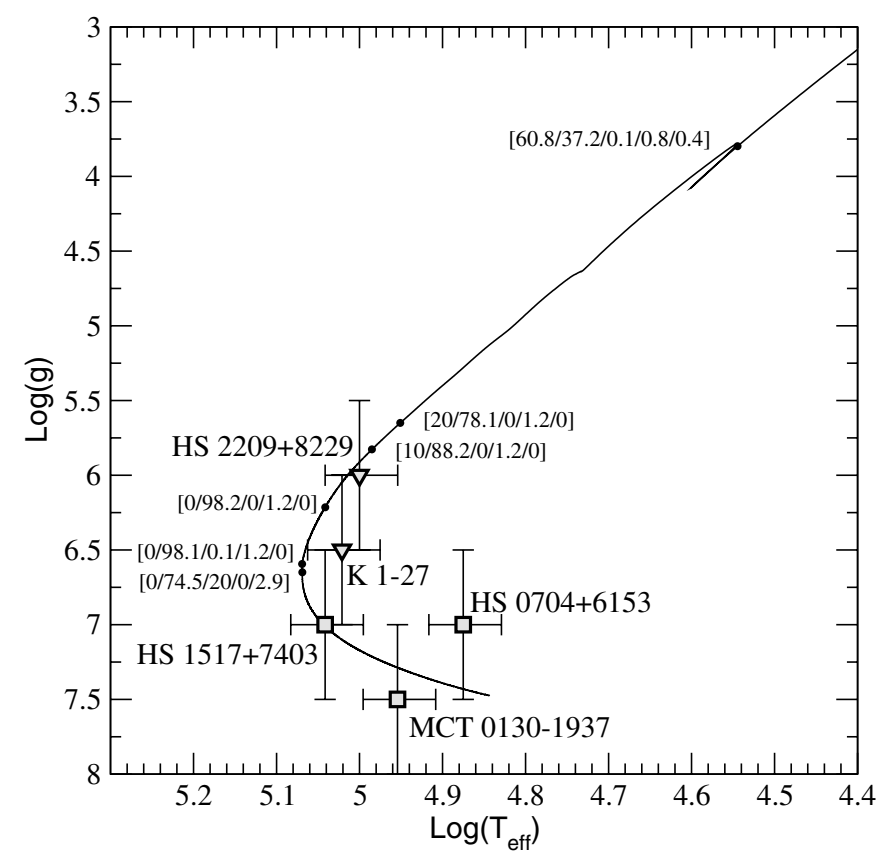

Fig. 8. Evolution in the $\log g-T_{\text {eff }}$ diagram for the $0.512 M_{\odot}$ remnant. Surface abundances $\left[\mathrm{H} / \mathrm{He} /{ }^{12} \mathrm{C} /{ }^{14} \mathrm{~N} /{ }^{12} \mathrm{O}\right]$ diplayed by the model are given at certain points (black dots) for comparison with the abundances displayed by the low mass $\mathrm{O}(\mathrm{He})$ stars and the He-enriched PG 1159 stars (triangles and square symbols, respectively).

one additional sequence for an initially $1 M_{\odot}$ star, but changing mass loss rates so that it undergoes its first thermal pulse while it was departing from the AGB (so its first thermal pulse is a LTP). This situation is more likely to occur in low-mass star progenitors (see Blöcker 1995). The resulting $0.512 M_{\odot}$ remnant is shown in Figs. 3 and 6 (post early AGB sequence). As can be seen this model can account for the location of many of the "redder" PG 1159 stars. However due to the low intensity of the first thermal pulses in low-mass stars no strong dredge-up is found and thus the sequence displays a normal surface abundance composition after the LTP. We analyzed the possibility that the model can turn into a PG 1159 due to mass loss. This is an important point as the model remains in the red giant region (where strong mass loss episodes are suppossed to occur) for an unusually long period of time after the LTP. To this end we follow the evolution using a mass loss rate of $10^{-6} M_{\odot} / \mathrm{yr}$ if $\log \left(T_{\text {eff }}\right)<3.8$ and $10^{-8} M_{\odot} /$ yr if $\log \left(T_{\text {eff }}\right)>3.8$. We find that such mass loss rates after the LTP can indeed turn the star into a PG 1159. The fact that the model remains in the red giant region for a long period of time is due to the several He-subflashes it experiences after the first thermal pulse (these sub-flashes are typical of the first thermal pulses of low-mass stars). As only one thermal pulse has happened, the final $\mathrm{He} / \mathrm{C} / \mathrm{O}$ abundances of this model (73/21/3) are very different from typical PG 1159 models. Also as no vigorous $\mathrm{H}$ burning took place, the star is also ${ }^{14} \mathrm{~N}$-deficient. The three He-enriched (and ${ }^{14} \mathrm{~N}$-deficient) objects in the non-pulsating sample of Dreizler \& Heber (1998) ${ }^{11}$ are located close to this track in the $\log g-T_{\text {eff }}$ diagram and show a very similar composition. Indeed we think that the mentioned correlation between $\mathrm{He}$ and ${ }^{14} \mathrm{~N}$ may reflect that the three nonpulsators with higher He-enriched surface abundances are the

11 These are HS 0704+6153, HS $1517+7403$ and MCT 0130-1937 $(\mathrm{He} / \mathrm{C} / \mathrm{O}=69 / 21 / 9,84 / 13 / 2,74 / 22 / 3$ respectively, Miksa et al. 2002).

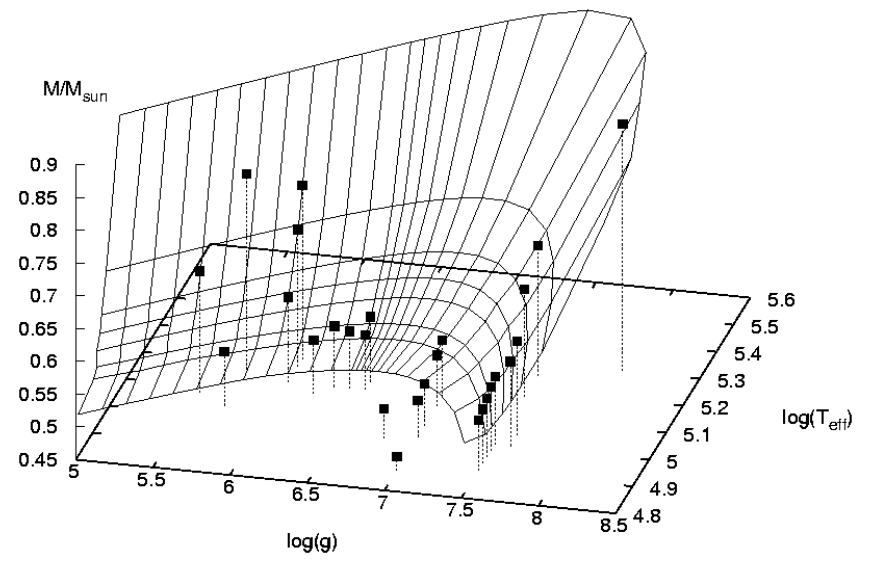

Fig. 9. Location of the sequences in the $g-T_{\text {eff }}-M$ space. Black squares show the derived location for real PG 1159 stars by adopting the $T_{\text {eff }}-g$ values presented by Werner \& Herwig (2006).

offspring of low mass stars that never reached the TP-AGB and suffered their first thermal pulse as a LTP.

In Fig. 8 we show the location of the $0.512 M_{\odot}$ sequence in the $\log g-T_{\text {eff }}$ diagram. Additionally the surface abundances $\left[\mathrm{H} / \mathrm{He} /{ }^{12} \mathrm{C} /{ }^{14} \mathrm{~N} /{ }^{12} \mathrm{O}\right]$ displayed by the model are given for certain points during its evolution. The first point (at $\log T_{\text {eff }} \approx$ 4.6) denotes the moment in which surface abundances start to change as a consequence of mass loss. At this point layers in which partial H-burning has happened are shown by the model. Abundances continue to change until the small He-buffer (similar to that of case 3 in Iben 1995) appears at the surface. At this moment, and until the He buffer is eroded by mass loss, the surface abundance of the models is of $98 \% \mathrm{He}$ and $1.2 \%{ }^{14} \mathrm{~N}$. These abundances are (surprisingly) similar to those exhibited by $\mathrm{K} 1-27$, which is located in the same region (see Fig. 8) as the model in the $\log g-T_{\text {eff }}$ diagram. Finally, when the He-buffer is removed by additional mass loss the stars diplay surface abundances typical of the He-enriched PG 1159. This happens while the model passes through the region (in the $\log g-T_{\text {eff }}$ diagram) in which He-enriched PG 1159 are located. Although the exact locus in the $\log g-T_{\text {eff }}$ diagram in which the star will show $\mathrm{O}(\mathrm{He})$ - or He-enriched- surface abundances depends on the exact value of the mass loss rate (which has been set somewhat artificially in this work), this numerical experiment shows that it is possible (with typical mass loss rates) that the low mass $\mathrm{O}(\mathrm{He})$ stars and the He-enriched-PG 1159 form an evolutionary sequence.

\section{Derivation of new spectroscopic masses}

By adopting the new tracks we have derived the theoretical locus of the models in the $g-T_{\text {eff }}-M$ space. We have done this by adopting a linear interpolation (i.e. by fitting a plane) between selected points along the theoretical tracks ${ }^{12}$. Once the theoretical surface in the $g-T_{\text {eff }}-M$ space was derived we calculated spectroscopic masses by adopting the $T_{\text {eff }}-g$ values presented by Werner \& Herwig (2006). In Fig. 9 we show both the theoretical surface and the derived parameters for real PG 1159 stars. The resulting spectroscopic masses are shown in Table 2.

\footnotetext{
12 Prior to this we tried a global least square polynomial fitting but it was inappropriate.
} 
Table 2. Masses derived from the new tracks presented in this work by adopting the values of $T_{\text {eff }}$ and $g$ from Werner \& Herwig (2006). Previous masses, which are based on old post-AGB tracks, have been taken from Werner \& Herwig (2006). Asterisks denote masses that have been derived by linear extrapolation and that are consequently more uncertain than the others. The new mean mass for the PG 1159 sample is $0.573 M_{\odot}, 0.044 M_{\odot}$ lower than previous mean mass. All masses are in $M_{\odot}$.

\begin{tabular}{cccc}
\hline \hline Star & $\begin{array}{c}\text { Previous } \\
\text { masses }\end{array}$ & $\begin{array}{c}\text { New } \\
\text { masses }\end{array}$ & $\Delta M$ \\
\hline H 1504+65 & 0.89 & 0.83 & -0.06 \\
RX J0122.9-7521 & 0.72 & 0.65 & -0.07 \\
RX J2117.1+3412 & 0.70 & 0.72 & 0.02 \\
HE 1429-1209 & 0.67 & 0.66 & -0.01 \\
PG 1520+525 & 0.67 & 0.61 & -0.06 \\
PG 1144+005 & 0.60 & 0.55 & -0.05 \\
Jn 1 & 0.60 & 0.55 & -0.05 \\
NGC 246 & 0.72 & 0.75 & 0.03 \\
PG 1159-035 & 0.60 & 0.54 & -0.06 \\
NGC 650 & 0.60 & 0.54 & -0.06 \\
Abell 21=Ym29 & 0.58 & 0.53 & -0.05 \\
K 1-16 & 0.58 & 0.54 & -0.04 \\
Longmore 3 & 0.59 & 0.54 & -0.05 \\
PG 1151-029 & 0.60 & 0.58 & -0.02 \\
VV 47 & 0.59 & 0.53 & -0.06 \\
HS 2324+3944 & 0.58 & 0.53 & -0.05 \\
Longmore 4 & 0.65 & 0.63 & -0.02 \\
SDSS J001651.42 & 0.65 & 0.63 & -0.02 \\
SDSS J102327.41 & 0.65 & 0.59 & -0.06 \\
PG 1424+535 & 0.57 & 0.51 & -0.06 \\
HS 1517+7403 & 0.57 & 0.51 & -0.06 \\
Abell 43 & 0.59 & 0.53 & -0.06 \\
NGC 7094 & 0.59 & 0.53 & -0.06 \\
SDSS J075540.94 & 0.62 & 0.58 & -0.04 \\
SDSS J144734.12 & 0.62 & 0.58 & -0.04 \\
SDSS J134341.88 & 0.62 & 0.58 & -0.04 \\
SDSS J093546.53 & 0.62 & 0.58 & -0.04 \\
SDSS J121523.09 & 0.62 & 0.58 & -0.04 \\
HS 0444+0453 & 0.59 & 0.55 & -0.04 \\
IW 1 & 0.56 & $0.50 *$ & -0.06 \\
Sh 2-68 & 0.55 & $0.50 *$ & -0.06 \\
PG 2131+066 & 0.58 & 0.55 & -0.03 \\
MCT 0130-1937 & 0.60 & 0.54 & -0.06 \\
SDSS J034917.41 & 0.60 & 0.54 & -0.06 \\
PG 1707+427 & 0.59 & 0.53 & -0.06 \\
PG 0122+200 & 0.58 & 0.53 & -0.05 \\
HS 0704+6153 & 0.51 & $0.47 *$ & -0.04 \\
\hline & & & \\
\hline
\end{tabular}

\section{Conclusions}

In this paper we have presented full evolutionary calculations for to post-AGB PG 1159 stars. In our calculations, the complete evolutionary stages of PG 1159 progenitors having a wide range of initial stellar masses have been considered, particularly the evolution through the very late thermal pulse (VLTP) and late thermal pulse (LTP) that occur following the departure from the AGB. The location of our PG 1159 tracks in the $\log g-T_{\text {eff }}$ diagram and their comparison with previous calculations as well as the resulting surface composition have been discussed. Our models are generally hotter than previous models of similar masses that do not display an efficient third dredge up during the TP-AGB, but are similar to recent models that include $\mathrm{OV}$ (and in which an efficient third dredge up is found during the TP-AGB) and other similar physical assumptions. This seems to reinforce the idea that the theoretical $\log g-T_{\text {eff }}$ diagram is sensitive to the previous evolution on the AGB.
Our results reinforces the idea that the different abundances of ${ }^{14} \mathrm{~N}$ observed on the surface of those PG 1159 stars with undetected hydrogen is an indication that the progenitors of these stars evolved through VLTP episodes, where most of the hydrogen content of the remnant is burnt, or late thermal pulses where hydrogen is not burnt but instead diluted to very low surface abundances.

We have also derived isochrones from our PG 1159 models aimed at discussing the presence of planetary nebulae in connection with the location of their central stars in the $\log g-T_{\text {eff }}$ diagram and their corresponding evolutionary ages. We infer that the progenitors of some PG 1159 stars (particularly NGC 650, VV47, IW, and PG 1520+525) might not have experienced a VLTP episode, in agreement with the correlation between the presence of ${ }^{14} \mathrm{~N}$ and pulsating PG 1159s (Dreizler \& Heber 1998). We also discuss the correlation between the presence of planetary nebulae and the ${ }^{14} \mathrm{~N}$ abundance as another indicator that ${ }^{14} \mathrm{~N}$-rich objects come from a born again episode while ${ }^{14} \mathrm{~N}$-deficient from a LTP.

Also, based on the new models presented here, we derived new values for spectroscopic masses. We find that the new masses are systematically lower and consequently at variance with those derived from asteroseismology. Although typical uncertainties in spectroscopic masses, and also uncertainties in astroseismological masses (as they are model- and methoddependent, Quirion et al. 2004; Corsico \& Althaus 2006), prevent us from drawing conclusions this difference may be indicating that the microphysics or the previous evolution (as for example the number of thermal pulses on the AGB) may be somewhat different than adopted in this work. Both improved determinations in $g$ and $T_{\text {eff }}$ for PG 1159 stars and the use of realistic PG 1159 models in asteroseismological analysis are needed in order to solve this problem.

Finally, we discuss the possibility that the He-enriched PG 1159 stars can be represented by very low mass objects, the progenitors of which abandoned the AGB shortly before the occurrence of the first thermal pulse. These progenitors experience their first thermal pulse as a LTP. We find that moderate mass loss rates operating during the evolution through the red giant region after the LTP could turn these objects into H-deficient ones, but with high surface helium abundances and a deficiency in nitrogen. In particular, we find that the surface abundances of HS 0704+6153, HS 1517+7403 and MCT 0130-1937, three helium enriched objects in the sample of Dreizler \& Heber (1998), can be reproduced within this scenario. Also our numerical experiment shows that this scenario provides a possible explanation for the existence of $\mathrm{O}(\mathrm{He})$ stars of low mass, linking them as the immediate ancestors of the He-enriched PG 1159.

Tabulations of $T_{\text {eff }}, L_{\star}$ and $g$ for the tracks presented in this work are freely available at our URL: http://www. fcaglp. unlp.edu.ar/evolgroup

Acknowledgements. We warmly acknowledge our referee (K. Werner) for his comments and suggestions which strongly improve the paper. We thank A. Córsico for a careful reading of the manuscript and A. Serenelli for sending us the post-core-helium-flash $1 M_{\odot}$ model. Part of this work has been supported by the Instituto de Astrofísica La Plata.

\section{References}

Alexander, D. R., \& Ferguson, J. W. 1994, ApJ, 437, 879

Althaus, L. G., Serenelli, A. M., Panei, J. A., et al. 2005a, A\&A, 435, 631 
Althaus, L. G., Miller-Bertolami, M. M., Córsico, A. H., García-Berro, E., \& Gil-Pons, P. 2005b, A\&A, 440, L1

Asplund, M., Lambert, D., Pollaco, D., \& Shetrone, M. 1999, A\&A, 343, 507 Blöcker, T. 1995, A\&A, 299, 755

Blöcker, T. 2001, Ap\&SS, 275, 1

Córsico, A. H., \& Althaus, L. G. 2006, A\&A, in press

Dreizler, S., \& Heber, U. 1998, A\&A, 334, 618

Fujimoto, M. Y. 1977, PASJ, 29, 331

Gautschy, A., Althaus, L. G., \& Saio, H. 2005, A\&A, 438, 1013

Hajduk, M., Zijltra, A., Herwig, F., et al. 2005, Science, 308, 231

Herwig, F. 2000, A\&A, 360, 952

Herwig, F. 2001, Ap\&SS, 275, 15

Herwig, F., Blöcker, T., Langer, N., \& Driebe, T. 1999, A\&A, 349, L5

Herwig, F., Freytag, B., Hueckstaedt, M., \& Timmes, F. 2006, ApJ, preprint

Iben, I. Jr. 1995, Phys. Rep., 250, 1

Iben, I. Jr., \& MacDonald 1995, Lect. Notes Phys. (Berlin: Springer-Verlag), 443,48

Iben, I. Jr., Kaler, J. B., Truran, J. W., \& Renzini, A. 1983, ApJ, 264, 605
Iglesias, C. A., \& Rogers, F. J. 1996, ApJ, 464, 943

Iglesias, C. A., Wilson, B. G., Rogers, F. J., et al. 1995, ApJ, 445, 855

Lawlor, T. M., \& MacDonald, J. 2003, ApJ, 583, 913

Miksa, S., Deetjen, J. L., Dreizler, S., et al. 2002, A\&A, 389, 953

Miller Bertolami, M. M., Althaus, L. G., Serenelli, A. M., \& Panei, J. A. 2006, A\&A, 449, 313

Quirion, P. O., Fontaine, G., \& Brassard, P. 2004, ApJ, 610, 436

Rauch, T., Dreizler, S., \& Wolff, B. 1998, A\&A, 338, 651

Rauch, T., Reiff, E., Werner, K., et al. 2004 [arXiv: astro-ph/0410698]

Saio, H., \& Jeffery, C. S. 2002, MNRAS, 333, 121

Schönberner, D. 1979, A\&A, 79, 108

Schönberner, D., \& Blöcker, T. 1992, Lect. Notes Phys. (Berlin: SpringerVerlag), 401, 305

Werner, K. 2001, Ap\&SS, 275, 27

Werner, K., \& Herwig, F. 2006, PASP, in press

Werner, K., Dreizler, S., Heber, U., \& Rauch, T. 1998, ASP Conf. Ser., 135, 130

Wood, P. R., \& Faulkner, D. J. 1986, ApJ, 307, 659 